- By 2010, large pharmaceutical companies were also losing their appetite for RNAi, severing collaborations and ending internal research programmes. "By and large, big pharma left RNAi for dead," says Fambrough. Safety concerns dealt the field another blow in 2016, when Alnylam abandoned one of its leading RNAi programmes after finding a possible link to patient deaths in a clinical trial (see 'Ups and downs').

But gradually, some RNAi companies began to iron out the kinks in their delivery systems. Alnylam experimented with a number of delivery routes and target organs, encasing some of its RNA molecules in fatty nanoparticles or chemically modifying the RNAs to help them survive the perilous journey through the bloodstream.

RNAs protected in this way and injected into the bloodstream tended to accumulate in the kidneys and liver. This led the company to look at transthyretin, which is produced mainly in the liver. In a clinical trial in 225 people with hereditary transthyretin amyloidosis who showed signs of nerve damage, average walking speed significantly improved in those who received the treatment (D. Adams et al.

\section{UPS AND DOWNS}

The biotech firm Alnylam faced several setbacks before winning US government approval for its first RNA-interference drug.

2,000

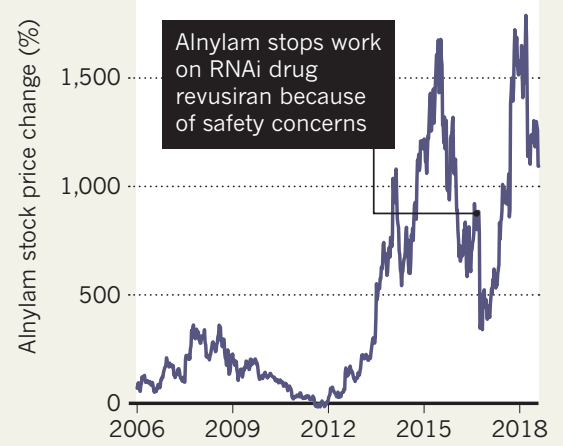

N. Engl. J. Med. 379, 11-21; 2018). Walking speed declined in the placebo group.

In the future, Alnylam and others will be able to move beyond the liver, says company co-founder Thomas Tuschl, a biochemist at Rockefeller University in New York City. Quark Pharmaceuticals of Fremont, California is testing RNAi therapies that target proteins in 웅 the kidneys and the eye. Alnylam is developing ways to target the brain and spinal cord, and Arrowhead Pharmaceuticals of Pasadena, California, is working on an inhalable RNAi treatment for cystic fibrosis.

"I've never been more optimistic about the future of RNAi," says Fambrough. "All of those tear-your-hair-out days were worth it to get to today."

Advances in RNA delivery might also benefit researchers who are developing gene-editing therapies based on the popular technique CRISPR-Cas9. That system uses a DNA-cutting protein called Cas9, which is guided to the desired site in the genome by an RNA molecule.

Like RNAi before it, CRISPR-Cas9 has become a common tool in genetics laboratories. But it might still face a difficult and lengthy path to the clinic. Much like ordinary drugs, RNAi therapies will break down over time; a gene edit, however, is intended to be permanent, which amplifies safety concerns.

"I hope they can do it more quickly than we did it, but I would not expect it to be so smooth," says Fambrough. "I wish them the best of luck." -

\section{BY JEFF TOLLEFSON}

$\mathrm{T}$ The US Environmental Protection Agency is making major changes to the way in which it evaluates chemicals for environmental and public-health effects. The latest push includes changes to chemicalsafety guidelines that place greater weight on industry-sponsored research, among other things, and is a part of efforts by US President Donald Trump's administration to reshape how the agency uses science to make decisions.

The Environmental Protection Agency (EPA) issued its chemical-assessment guidance in May, and is soliciting public comments until 16 August. The guidance contains changes dictating the kind of data that studies must include in order to be considered in the EPA's decision-making process. Researchers and environmental and public-health advocates say that the guidelines provide a non-peerreviewed alternative to the EPA's main system for conducting chemical reviews and calculating acceptable exposure limits. The agency is required by law to do these evaluations, but the guidance defines how officials conduct them. At stake are tens of thousands of chemicals destined for public use and governed by the 1976 Toxic Substances Control Act (TSCA).

The guidance dovetails with a rule proposed in April by then-EPA administrator Scott Pruitt, which, if finalized and implemented, would reduce the role of published scientific studies in decision-making across the agency. The changes also coincide with attacks on the EPA's core chemical-assessment programme, known as the Integrated Risk Information System (IRIS), by industry and Republican politicians over the past year.

In a statement to Nature, the EPA says the changes are meant to provide clear criteria to help determine the quality of the research used to evaluate chemicals - and that the guidance is a work in progress that can be revised in response to new information. But scientists say the process laid out by the EPA is at odds with established, peer-reviewed procedures for such assessments.
Jennifer Sass, a senior scientist at the Natural Resources Defense Council, an advocacy group based in New York City, suspects that the goals are to promote science from industry and change the calculations that the EPA uses to develop regulations and estimate safe exposure limits for chemicals.

\section{MEETING THE REQUIREMENTS}

The guidelines introduce many data reporting requirements - including statistical analyses that measure whether a study correctly identifies the presence of an effect - that are standard for industry-funded research. But because such criteria vary among peer-reviewed journals, many academic studies would be disqualified, says Tracey Woodruff, who led the development of a chemical-evaluation process at the University of California, San Francisco. "Only industry studies will survive."

The changes represent a major shift because they create a new system for chemicalrisk assessments under TSCA. Unlike 


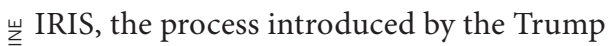
administration has not been peer reviewed, and yet it would allow agency officials to circumvent IRIS evaluations. Under former president Barack Obama, the EPA would have used IRIS to perform these reviews when considering regulations under TSCA.

The IRIS programme dates back to 1985 , but under the Obama administration, the EPA modernized and standardized its chemicalevaluation procedures to improve transparency and confidence in its health assessments. Woodruff says that the IRIS process is solid and that bypassing it would be a mistake.

"The TSCA office is deciding to ditch all of the experts and empirical methods that have been developed over the last 30 years for a method that appears to be based on their whim and personal opinion," she says.

But the EPA insists that the review process used in these chemical evaluations is intended to "comprehensively capture all available science".

Politicians in the US House of Representatives have also hammered IRIS, holding hearings questioning the quality and validity of the programme's assessments. The political manoeuvring parallels efforts from industry to bypass scientific reviews of certain chemicals.

One plant in LaPlace, Louisiana, makes the chemical chloroprene for the Tokyo-based company Denka. Chloroprene is used to make neoprene, a synthetic rubber integral to products such as wetsuits. A 2010 IRIS evaluation and subsequent government studies suggested

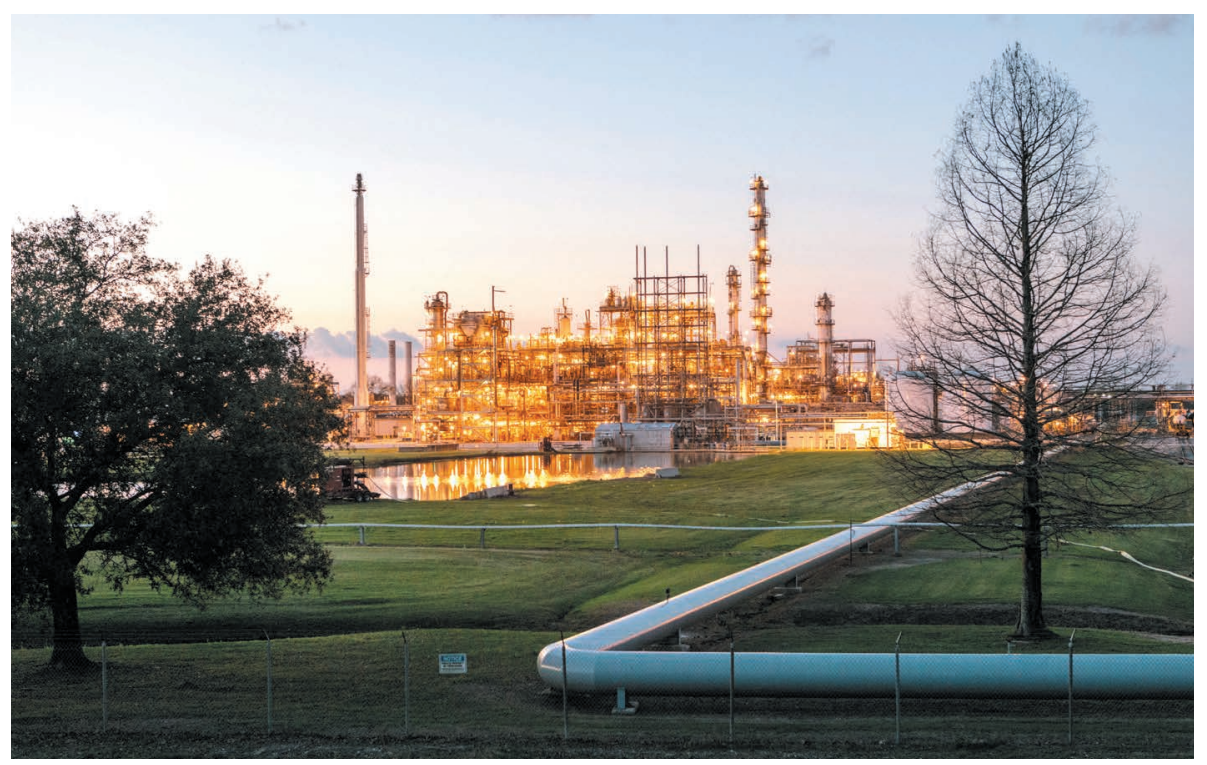

A chemical produced in a US-based plant is part of a challenge to a government safety programme.

that chloroprene exposure levels in LaPlace were high enough to increase cancer risk in some areas of the city. Denka challenged that ruling last year, arguing that the assessment was incorrect. The company lost its challenge in January but has since appealed against that ruling. A panel appointed by the EPA leadership will now consider the appeal.

Denka has argued to its political allies that reducing chloroprene emissions would be too expensive, says Karl Brooks, a former EPA official who last year served as a consultant in a lawsuit filed by LaPlace residents against Denka. That's a potentially dangerous development, he says, because IRIS assessments are meant to focus on the health effects of chemicals - not the economic challenges that a company might face as a result of the core science.

Researchers fear that the chloroprene case represents yet another strategy for companies seeking relief from the burdens of regulations: challenge the science and, when that fails, appeal to friendly politicians and political appointees.

\section{LHC teams turn to brute-force hunt}

\section{World's most-powerful particle collider is using a fresh approach to find evidence of 'new' physics.}

\section{BY DAVIDE CASTELVECCHI}

A once-controversial approach to particle physics could soon have an increased role at the Large Hadron Collider (LHC). The LHC's major ATLAS experiment has officially thrown its weight behind the method - an alternative way to hunt through the reams of data created by the machine - as the collider's best hope for detecting behaviour that goes beyond the standard model of particle physics, because conventional techniques have so far come up empty-handed.

So far, almost all studies at the LHC - at CERN, Europe's particle-physics laboratory near Geneva, Switzerland - have involved 'targeted searches' for signatures of favoured theories. The ATLAS collaboration now describes its first all-out 'general' search of the detector's data - a kind of brute-force approach — in a preprint posted last month and submitted to European Physics Journal C (ATLAS Collaboration. Preprint at https://arxiv.org/abs/1807.07447v1; 2018). Another major LHC experiment, CMS, is working on a similar project.

"My goal is to try to come up with a really new way to look for new physics" - one driven by the data rather than theory, says Sascha Caron of Radboud University Nijmegen in the Netherlands, who has led the push for the approach at ATLAS. General searches are to the targeted ones what spell-checking an entire text is to searching for a particular word. These broad searches could realize their full potential soon, when combined with increasingly sophisticated artificial-intelligence (AI) methods.

LHC researchers hope that the methods will lead them to their next big discovery something that hasn't happened since the detection of the Higgs boson in 2012, which put in place the final piece of the standard model. The model describes all known subatomic particles, but physicists suspect that there is more to the story - the theory doesn't account for dark matter, for instance. But big experiments such as the LHC have yet to find evidence for this behaviour. That means it's important to try new things, including general searches, says Gian Giudice, who heads CERN's theory department and is not involved in any of the experiments. "This is the right approach, at this point."

\section{COLLISION COURSE}

The LHC smashes together millions of protons per second at colossal energies to produce a profusion of decay particles, which are recorded by detectors such as ATLAS and CMS. Many different types of particle interaction can produce the same debris. For example, the decay of $>$ 